\title{
A Conversation Between David Collingridge and Henryk Barthel
}

\author{
David Collingridge ${ }^{1}$ and Henryk Barthel ${ }^{2}$ \\ ${ }^{I}$ The Lancet Oncology, London, United Kingdom; and ${ }^{2}$ Leipzig University, Leipzig, Germany
}

$\mathbf{T}$ his month, Johannes Czernin, MD, editor-in-chief of The Journal of Nuclear Medicine, asked Henryk Barthel, MD, PhD, a professor in the Klinik und Poliklinik für Nuklearmedizin at the University of Leipzig (Germany), to talk with David Collingridge, $\mathrm{PhD}$, editor-in-chief of The Lancet Oncology and publishing director of The Lancet specialty journals based in London, U.K. He is also a Clinical Associate Professor of Radiation Medicine at Hofstra/Northwell Health (Lake Success, NY). His doctorate in tumor biology was earned at University College London (U.K.). Before moving into medical publishing in 2001, he was a radiobiologist with an interest in the tumor microenvironment and the ways in which modification and dynamic monitoring of tumor physiology could be exploited to improve responsiveness to radiotherapy. His research activities at Yale University (New Haven, CT) and at Imperial College School of Medicine/Hammersmith Hospital (London, U.K.) involved interventions modifying tumor hypoxia, blood flow, and immune biology; and imaging with MR, SPECT, and PET to aid manipulation of radiotherapeutic response. Under Dr. Collingridge's leadership, The Lancet Oncology has developed into a world-leading clinical oncology journal. In recent years, he has overseen the launch of 14 additional specialty journals in The Lancet portfolio, with plans for more in the coming years.

Dr. Barthel: David, we have known each other for a long time, and, as I consider you a good friend, I hope it is OK if we keep the personal form of address.

Dr. Collingridge: Absolutely fine, Henryk.

Dr. Barthel: With your decision in 2001 to give up doing your own research and, instead, to publish research done by others, you have a very interesting scientific career. Tell me more about your motivations to initially be a cancer researcher but then to leave that world for a second career in publishing?

Dr. Collingridge: I was first attracted to cancer biology while at school. I was 15 or 16 years old at the time and had a really good biology teacher who made the classes interesting. I found myself fascinated by a (rather simplistic in hindsight) educational module on cancer. That interest stayed with me and fed into my choice of degree: biological sciences with specializations in genomics and microbiology, both of which are fundamental building blocks in cancer biology. This was followed with a $\mathrm{PhD}$ in tumor biology and, specifically, radiobiology. My PhD at the Gray Laboratory in London really shaped my long-term research interests, first at Yale and then at the Hammersmith Hospital. I was a translational radiobiologist in an era when translational science was not as in

COPYRIGHT @ 2020 by the Society of Nuclear Medicine and Molecular Imaging. vogue as today-but to me it was logical that there was a circular relationship between basic research in the lab, how it translated in patients, and subsequent cycling of those clinical learnings back into the lab to refine the basic research. My interests at the time focused on how to improve radiation response by modifying the tumor microenvironment and the ways in which imaging (MR and PET imaging) could be used to better individualize treatment and monitor outcomes to refine ongoing treatment. I was very

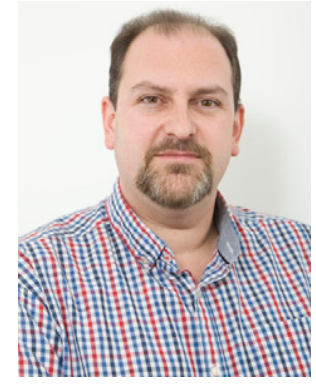

David Collingridge,
PhD lucky to work with some inspiring mentors during my research years: Professors Dai Chaplin, John Griffiths, Sara Rockwell, Jonathan Knisely, Eric Aboagye, and Pat Price, to name just a few. Under their guidance, I undertook research into in vivo measurement of hypoxia in animal tumor models, in patients in the clinic, and correlations between the two; research into several physiologic and pharmacologic radiosensitizers and the resultant radiobiologic impacts both in laboratory models and in the clinic; and development of novel MRI modalities and various new radiotracers for PET imaging of tumor metabolism, hypoxia, blood flow, and apoptosis. However, toward the end of my research career, I was more and more intrigued by the broader clinical implications of such research and more motivated by how to better disseminate the findings of research to actually shape and inform health systems. It was these motivating factors that led me to The Lancet, where I found a lot of like-minded people.

Dr. Barthel: Amazing how a good schoolteacher can inspire kids to pursue a science career-we need more of them! Before we talk about The Lancet, let us come back for a moment to your research in London. I had the great honor of joining Eric Aboagye and Pat Price's PET oncology research group at the Hammersmith Hospital in 2000, at a time when you were already doing interesting research there. What a great place full of interdisciplinary exchange between highly skilled and motivated scientists! We worked together on different tumor PET tracers, like ${ }^{18}$ F-FLT and the hypoxia tracer ${ }^{18}$ F-FETA. I learned so much from you, Eric, and the other scientists at that time. Was there something that made the Hammersmith Hospital special to you as well? Apart from the Speakeasy, the only pub I've ever seen on a hospital campus.

Dr. Collingridge: A lot has certainly changed in 20 yearsstaff social clubs at hospitals in the U.K. are (sadly) no longer commonplace, but I do recall multiple occasions putting the world to rights at the end of a long day of cell culture or tumor implantation! Joking aside, working at the Hammersmith Hospital in the 
PET Oncology Group had a lot of resonance for me. In the early 1950s, Professor Hal Gray-the person after which absorbed radiation dose is named-did some of the early pioneering work in radiobiology at the Hammersmith, before moving to Mount Vernon Hospital in north London to set up his own radiobiology institute, the Gray Laboratory. As a PhD alumnus of the Gray Laboratory, the opportunity for me to work at the Hammersmith completed an interesting historical arc. It was a fascinating time, we had just installed the world's first dedicated small-animal PET scanner, under Professor Terry Jones's leadership, which opened up the possibility to move beyond traditional biodistribution analyses of new PET tracers to look more completely at the correlation with in vivo imaging and the tracer's role in therapeutics. During this time, you and I worked on several novel tracers, as you mention, but I also had interests in ${ }^{124}$ I-AnnexinV for apoptosis imaging, ${ }^{124} \mathrm{I}$-VG76e (an iodolabeled vascular endothelial growth factor antibody for vascular imaging), and an early idea to develop a radiotracer to measure tumor-infiltrating macrophages, which I was becoming more and more convinced held the key to many of the fundamentals of tumor radiobiology. It was a great lab to work in, with a lot of freedom to explore new ideas. Being based on the hospital campus, we also had the possibility of working in a translational environment where new tracers and ideas could potentially be trialed in patients.

Dr. Barthel: Exciting stuff! Tell me more about your experiences with tumor hypoxia PET tracers. I was always intrigued by the increasingly means immunotherapy), rather than increasing reliance on a single intervention, however advanced that might be.

Dr. Barthel: I get your point. If we talk about the acceptance of new diagnostic concepts to potentially improve individualized therapy, it must translate into improved patient outcomes. This brings me to the theranostic principle, the currently most exciting clinical care and research area that drives the nuclear medicine field. Theranostic tracer pairs are in clinical use to handle thyroid, neuroendocrine, and prostate malignancies. New theranostic pairs, potentially to deal with a much wider range of tumor entities, are under development. What is your take on the utility of this principle, especially with regard to individualized medicine?

Dr. Collingridge: This is really where the future of nuclear medicine is heading, in my opinion. Theranostics has enormous potential as a treatment modality. Radiolabeled prostate-specific membrane antigen, for example, is looking like a step-change in the way in which we treat prostate cancer. The early results in metastatic disease are truly remarkable. Theranostics provides an opportunity to provide systemic radiotherapy on one hand and a more precise medical oncology on the other, depending on which radioconjugate is being used. However, even more important, the evolution of nuclear medicine into a theranostics specialty (and I include interventional radiology in this definition) could have a much broader impact on the politics of medicine and health services. Nuclear medicine could see itself redefined as front and center in

\section{"In this era of fake news, minority opinions being unduly amplified via social media, and an ability to post anything online irrespective of facts, journals must reassert their claim to be trusted beacons for evidence and opinion."}

unique concept behind these tracers, namely the idea of visualizing the deficiency of a target parameter (oxygenation) in positive image contrast. What is your explanation for the fact that this concept has not managed (so far) to enter clinical care, for example, to improve dose painting in radiation oncology?

Dr. Collingridge: This is a very good question! Although under controlled conditions, it is very easy to demonstrate the importance of oxygen in enhancing radiation damage during radiotherapy, the challenge clinically (and in vivo in the lab to some extent) is the added complexity of unpredictable blood flow, which affects the ability to saturate a tumor with a radiosensitizing drug or hyperoxygenated blood; the ability of tumors to survive and thrive in anaerobic conditions; the high mutational load and carcinogenic adaptation; the underlying genomic heterogeneity in the tumor bed and its effect on radiosensitivity; the role of the immune system, cell-cell crosstalk, and various intracellular and extracellular components involved in cell metabolism and survival; and, finally, the inability to use radiation to target metastatic spread of a tumor. Taken together, although dynamic imaging of tumor hypoxia and dose adaptation is possible and can drive enhanced cell kill in a primary cancer, it is often undone by the wider biology at play, meaning the overall therapeutic enhancement is limited because of recurrence, metastases, or genetic adaptation of the primary tumor. Ultimately, patient survival is arguably just not improved enough to justify the added technical complexity and cost. This has led research in the direction of putting more emphasis on improving the delivery of radiation to elicit more localized cell kill and fewer adverse events, coupled with use of systemic treatments (which patient care-not just a supportive specialty on which oncologists and other specialists refer to for help in guiding the management of their patients. Nuclear physicians could become the primary coordinator of care in some major settings. This would be a big departure in the way health services are organized currently.

Dr. Barthel: I think this is a really exciting prediction, and I agree that there is great potential for our field to take a more leading role by using theranostic approaches in future cancer patient care. It works perfectly for thyroid cancer patients in many European countries, so why not in other malignancies and in other parts of the world? Such a paradigm shift in nuclear medicine would, on the other hand, need a massive rethinking of many aspects of our specialty, like how we train future doctors, what our general goals and priorities are, how we cooperate with other specialties, what additional management skills we will need to acquire, etc. Where should we start?

Dr. Collingridge: This is indeed a multifaceted issue. The starting point is ramping up current research, globally, to develop a range of theranostics that are ultimately proven to be more effective for major cancers than existing therapies. This will create momentum, dictating the central role for nuclear medicine in treatment pathways, which would drive a lot of change in itself. An additional step, though, is cultural: creating institutional treatment guidelines based on evidence that ensures that patients' treatments are not determined by who sees them first. This is a common problem in oncology - the trajectory of a treatment plan is often influenced by who saw the patient first, even if that plan is not the best option. Finally, many health systems need to adapt to properly effective hub-and-spoke referral models that are patient-centric 
and affordable. Layered around all of this are many other issues, of course, as you rightly highlight, including service commissioning and financing, education and training, day-to-day logistics, and so on. But it does feel that we are on the cusp of a major sea change in the role of nuclear medicine, not only in cancer care but also in other major medical disciplines.

Dr. Barthel: I agree, David, molecular dementia imaging, my current field of research, might become another big thing, reflecting the new way of thinking about Alzheimer disease as a biologic construct rather than a syndrome, with the first anti-Alzheimer drug targeting amyloid pathology currently under review by the Food and Drug Administration.

Let's change gears to another area I am extremely excited to hear about. I would like to talk about your activities at The Lancet Oncology in bringing people together to address major unmet needs in medicine on a global scale. Can you give us some insights into these activities?

Dr. Collingridge: For the past 10 years, The Lancet Oncology has run a large program focused on global oncology. Through an international advocacy program, we have been trying to map out the inequalities and inequities in health systems worldwide, highlighting deficiencies in all aspects of cancer care, health policy, structural organization, and leadership. Because The Lancet journals have no formalized affiliations, it gives us a unique and neutral platform to bring together thought leaders from across different disciplines and organizations to offer solutions to those barriers that hinder the provision of high-quality cancer control. We approach these issues via several different types of initiatives, but our flagship approach is called the "Commission." This is a project that results in a large white paper spelling specific actions but starts with us identifying and working with global leaders, often over many years, on a specific inequality in cancer care.

Dr. Barthel: Wonderful. Your journal is not only after medical developments by publishing and discussing cutting-edge research, you are also contributing to shaping the future. Does this great initiative also deal with the cancer diagnostics field?

Dr. Collingridge: Yes, absolutely. One example that will be of interest to The Journal of Nuclear Medicine (JNM) readers is our upcoming Commission on cancer imaging that will map out the landscape in low-to-middle income countries and present an investment case on how to build an imaging service in these lowerresourced nations. Access to affordable imaging is, of course, an essential component of any effective cancer health care system. This Commission, led by Professor Hedvig Hricak from Memorial Sloan Kettering Cancer Center (New York, NY) and involving a large number of key opinion leaders worldwide, has already been endorsed by all the major radiology and nuclear medicine societies globally and will be launched in a plenary session at this year's World Cancer Congress in Oman in October. In addition to support from societies, this Commission has also been strongly supported by the International Atomic Energy Agency, which will help in implementation later on, and by the Union for International Cancer Control, who have been long-term supporters of many of our Commissions.

Dr. Barthel: Great! Will this Commission also specifically investigate the current situation for nuclear medicine imaging technology?

Dr. Collingridge: As this Commission is one of our global health-focused projects, it will not be looking specifically at the current state of the art in nuclear medicine or future technologic developments per se. However, to understand how to best invest in building or augmenting an imaging service in a country that currently has limited or no facilities, it is important to understand the current direction of travel to ensure that the recommended best buys and working practices are future-proof and that any new service is not providing substandard or unsafe care nor will it be outmoded before a return on the investment is realized.

Dr. Barthel: This sounds like a very good plan to me. I imagine there are massive gaps in medical imaging supply in many parts of our world. What usually follows in these kinds of initiatives once the current state is evaluated and documented? Will The Lancet Oncology also monitor the next steps taken and check to see whether the situation improves?

Dr. Collingridge: No single Commission is the same, but the more successful ones have seen societies and nongovernmental organizations (NGOs) adopt the findings to shape their own initiatives and programs on the basis of the targets and ambition presented in the reports. As a journal we continue to promote the findings of each Commission via conferences, events, governmental meetings, social media, spin-off programs with other partners, and the like, for many years. Then we typically reconvene the leads from the original Commission group to monitor progress, highlighting what has been achieved, what remains to be done, and whether something new has emerged in the intervening time that requires new thinking and analysis. These subsequent progress reports can take many forms, from a single article or a small series of papers to a new Commission.

Dr. Barthel: Another question that interests me is how "affordable" is defined with regard to imaging technology within a health care system. In cancer patients, for example, we nuclear medicine imagers are often confronted with the allegation of being too expensive. I think this is unfair, because such judgments do not consider the cost saving related to improved staging and improved individualized treatment via the imaging we use. In the end, cost-intensive molecular imaging might become an economically attractive field of investment, even for medium-or low-income countries. Will this aspect also be investigated by this Commission?

Dr. Collingridge: Very good point, Henryk. Affordability is a complex term not related to the absolute cost of providing a service. Wrapped up in the definition of affordability is value: how much value do we as society place on a particular product or service? At a national level, in a universal health system, this is also fundamentally a political decision. Cost often becomes a lesser concern if there is political buy-in. But you are absolutely right to raise the bigger picture in funding an imaging service. We have had similar debates in many of our other projects. Our radiotherapy Commission in 2015, for example, showed that a single dose of radiation is far more cost effective than any other medical intervention for cancer treatment, despite the large initial expenditure. Likewise, nuclear medicine involves a large capital investment coupled with long-term maintenance and running costs before consideration of the individual medical costs per patient. However, with any medical intervention it is important to look at the cost holistically, within the context of all other aspects of care: the cost saving brought about by tailoring and modifying treatments over time, the reduction in long-term health care by more effective upfront image-guided treatment, and the cross-benefits of commissioning a new service in all areas of the health system, not just the specialty under the current microscope. Installing a PET scanner does not just provide a service to the oncology department, for example. It is important to look at total value to the health system as a whole, not just the building and operational 
costs. The imaging Commission will take all of this into account to reach its verdict.

Dr. Barthel: I very much look forward to reading these publications in your journal. By the way, would it be possible for you to share with us JNM editors and our readers some of the secrets of success that have made The Lancet Oncology the leading journal in the field?

Dr. Collingridge: The short answer has been ensuring that the journal is not just mirroring the latest advances in oncology but also having an opinion and taking a leadership position on current issues of relevance to the oncology community worldwide. We also work hard to offer a personalized and fast service to our authors, readers, and reviewers by being truly contactable and open to different opinions and ideas, even if they might be contentious on occasion. And, finally, The Lancet family of journals is quite unique in operating in a highly collegial and collaborative way, which means authors can get multiple opportunities across the portfolio from a single inquiry, submission, or conversation. We certainly believe that collective action across our titles on certain issues is bigger than the sum of the individual parts.

Dr. Barthel: Very interesting and greatly appreciated! My final question is on where you see the medical science journal landscape heading in the future: Will traditional journals like yours and the JNM remain important in the coming years, given that there are new ways to publish medical research?

Dr. Collingridge: The publishing landscape is most definitely changing, and, to survive, journals cannot simply be repositories. There are many ways of distributing ideas without a traditional publisher. Journals need to be health activists challenging the establishment and providing a loud voice for the communities they serve to change health care for the better. Journals need to operate more like NGOs. Too often, changes are top-down and initiated by people with limited or narrow understanding, or by individuals influenced by their own biases. Journals can act as advocates for change, providing a vehicle for health professionals to influence policymakers on the basis of real-world knowledge and experience. In this era of fake news, minority opinions being unduly amplified via social media, and an ability to post anything online irrespective of facts, journals must reassert their claim to be trusted beacons for evidence and opinion. Without this the many great advances in medicine could be irrevocably undone.

Dr. Barthel: I cannot agree more on that point, David. Thanks so much for your time and for your willingness to share your thoughts. It's been truly a great pleasure talking to you. 